\title{
The deconfinement phase transition in the Hamiltonian approach to Yang-Mills theory in Coulomb gauge
}

\author{
Hugo Reinhardt* \\ Universität Tübingen, Institut für Theoretische Physik \\ Auf der Morgenstelle 14, 72076 Tübingen, Germany \\ E-mail: hugo.reinhardt@uni-tuebingen.de
}

Davide R. Campagnari and Jan Heffner

Universität Tübingen, Institut für Theoretische Physik

Auf der Morgenstelle 14, 72076 Tübingen, Germany

\begin{abstract}
The deconfinement phase transition of SU(2) Yang-Mills theory is investigated in the Hamiltonian approach in Coulomb gauge assuming a quasi-particle picture for the grand canonical gluon ensemble. The thermal equilibrium state is found by minimizing the free energy with respect to the quasi-gluon energy. At the deconfinement phase transition the gluon energy, being infrared divergent in the confined phase, becomes infrared finite in the deconfined phase, while the ghost form factor remains infrared divergent in the deconfined phase but its infrared exponent is approximately halved. Using the lattice results for the gluon propagator to fix the scale the deconfinement transition temperature is obtained in the range of 275 to $290 \mathrm{MeV}$.
\end{abstract}

Xth Quark Confinement and the Hadron Spectrum,

October 8-12, 2012

TUM Campus Garching, Munich, Germany

\footnotetext{
* Speaker.
} 


\section{Introduction}

Understanding the deconfinement phase diagram of QCD is one of the major challenges of particle physics. In this talk I will report on a description of the deconfinement phase transition at zero baryon density within the Hamiltonian approach to Yang-Mills theory in Coulomb gauge, extending the previously developed variational approach for the Yang-Mills vacuum [1] to finite temperatures. This approach has been quite successful in understanding the infrared properties of Yang-Mills theory: One finds a gluon energy which is infrared divergent [1] and a linearly rising quark potential [2], both being signals of confinement. In addition, one finds an infrared enhanced running coupling constant with no Landau pole [3], a perimeter law for the 't Hooft loop [4] and, within an approximate Dyson-Schwinger equation, an area law for the spatial Wilson loop [5]. Also the topological susceptibility was found in accord with the lattice data [6]. Given the success of this approach in the vacuum sector we can also expect a decent description of the deconfinement phase transition. The outline of my talk is as follows: I will first review the basic ingredients of the Hamiltonian approach to Yang-Mills theory in Coulomb gauge. Within this approach I will then study the grand canonical ensemble in a mean-field type approximation and investigate the deconfinement phase transition.

\section{Hamilton approach to Yang-Mills theory}

The Hamilton approach to Yang-Mills theory is based on the canonical quantization in Weyl gauge $A_{0}=0$, which yields the Hamiltonian

$$
H=\frac{1}{2} \int \mathrm{d}^{3} x\left(\Pi^{2}(x)+B^{2}(x)\right)
$$

where $\Pi(x)=-\mathrm{i} \delta / \delta A(x)$ is the canonical momentum operator. Due to the use of the Weyl gauge Gauss' law escapes the quantum equations of motion and has to be imposed as a constraint to the wave functional

$$
D \Pi \psi[A]=0 .
$$

Here $D$ denotes the covariant derivative in the adjoint representation of the gauge group. The operator in Gauss' law $D \Pi$ is nothing but the generator of (time-independent but space-dependent) gauge transformations and Gauss' law ensures that (in the absence of matter fields) the wave functional must be gauge invariant. To respect gauge invariance one can work with explicitly gauge invariant wave functionals, which has been pursued mainly in 2+1-dimensions but which becomes exceedingly involved in 3+1-dimensions. A more convenient way is to fix the gauge and resolve Gauss' law explicitly. For this purpose Coulomb gauge is convenient and after gauge fixing one finds the following Hamiltonian

$$
H=\frac{1}{2} \int \mathrm{d}^{3} x\left(J_{A}^{-1} \Pi(x) J_{A} \cdot \Pi(x)+B^{2}(x)\right)+H_{\mathrm{C}},
$$

where $J_{A}=\operatorname{Det}(-D \partial)$ is the Faddeev-Popov determinant and

$$
H_{\mathrm{C}}=\frac{g^{2}}{2} \int \mathrm{d}^{3} x \mathrm{~d}^{3} y J_{A}^{-1} \rho^{a}(x) J_{A} F^{a b}(x, y) \rho^{b}(y)
$$




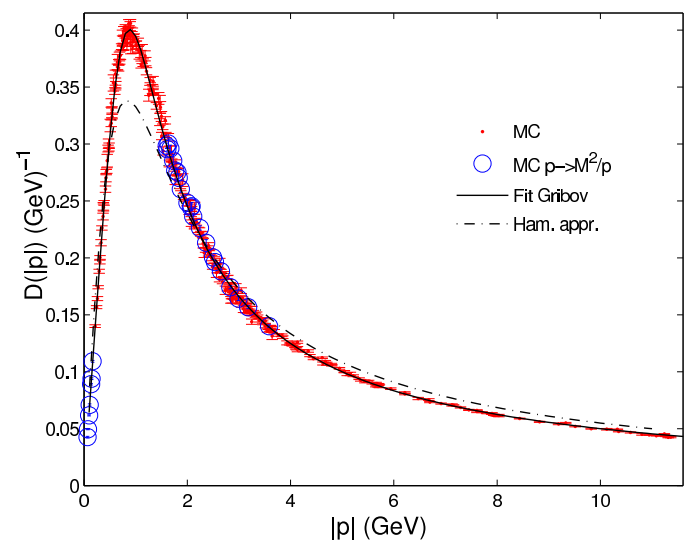

Figure 1: Comparison of the results of the variational calculation and lattice data for the gluon propagator.

is the so-called Coulomb Hamiltonian. Here, $\rho^{a}=-f^{a b c} A^{b} \Pi^{c}$ is the color charge density of the gluons. The gauge fixed Hamiltonian is highly non-local due to the presence of the Faddeev-Popov determinant and the Coulomb term $H_{\mathrm{C}}$. Although the gauge fixing gives rise to a more complicated (non-local) Hamiltonian it has the advantage that the gauge invariance has been taken care of once and for all and that after Coulomb gauge fixing any wave functional depending on the transversal gauge field only lies in the physical Hilbert space. Fortunately it turns out that in the gluon sector, in particular for the investigation of the infrared properties, the Coulomb term can be ignored, which I will do in the following.

With the Coulomb gauge fixed Hamiltonian (2.3) the Yang-Mills Schrödinger equation has been solved by a variational principle using Gaussian type ansätze for the vacuum wave functional. The approach developed in our group differs from previous attempts $[7,8]$ by the ansatz for the vacuum wave functional, by the full inclusion of the Faddeev-Popov determinant and by the renormalization. Our ansatz for the vacuum wave functional reads

$$
\psi[A]=J_{A}^{-1 / 2} \exp \left[-\frac{1}{2} \int A \omega A\right],
$$

where $\omega$ is a variational kernel, which is determined by minimizing the energy $\langle\psi|H| \psi\rangle$. The preexponential factor has the advantage that it cancels the Faddeev-Popov determinant in the scalar product of the Coulomb gauge fixed wave functionals. Furthermore, for this wave functional the gluon propagator is given by

$$
\langle A A\rangle=(2 \omega)^{-1},
$$

which identifies the Fourier transform of $\omega$ as quasi-gluon energy. Figure 1 shows the result of the variational calculation for the gluon propagator in comparison with the lattice data. As one observes, the cross feature of the lattice data are well reproduced. In particular, in the deep infrared regime our variational calculations agree perfectly with the lattice data. There are deviations in the mid-momentum regime, which can be substantially reduced by using non-Gaussian wave functionals, Ref. [9]. What is also remarkable is that the lattice data can be nicely fitted by Gribov's formula

$$
\omega(p)=\sqrt{p^{2}+M^{4} / p^{2}}
$$

with the mass $M=0.88 \mathrm{GeV}$. 


\section{The grand canonical ensemble}

Since the gluons have vanishing chemical potential their grand canonical ensemble is defined by the density operator

$$
D=\exp (-\beta H),
$$

where $H$ is the gauge fixed Hamiltonian (2.3) and $\beta=1 / k_{B} T$ is the inverse temperature. By means of the density matrix thermal averages are defined by

$$
\langle\ldots\rangle_{T}:=\frac{\operatorname{Tr}(D \ldots)}{\operatorname{Tr} D},
$$

where the trace can be calculated, in principle, in any complete basis. However, we will necessarily have to introduce approximations and then the choice of the basis matters. We will choose an optimal basis by exploiting the variational principle. Inspired by the zero temperature calculations, see Eq. (2.5), we will choose our basis in the form

$$
|\tilde{k}\rangle=\frac{1}{\sqrt{\operatorname{Det}(-D \partial)}}|k\rangle,
$$

where the states $|k\rangle$ are defined by the Fock basis

$$
|0\rangle, \quad a^{\dagger}(p)|0\rangle, \quad a^{\dagger}\left(p_{1}\right) a^{\dagger}\left(p_{2}\right)|0\rangle, \quad \ldots
$$

obtained by decomposing the gauge field in terms of creation-annihilation operators

$$
A(k)=\frac{1}{\sqrt{2 \omega(k)}}\left(a(k)+a^{\dagger}(-k)\right)
$$

with a so far arbitrary kernel $\omega(k)$ and the vacuum state, being defined by $a(k)|0\rangle=0$, is the Gaussian

$$
\langle A \mid 0\rangle=\exp \left(-\frac{1}{2} \int A \omega A\right)
$$

Inserting this state into Eq. (3.3) we find that the state $|\tilde{0}\rangle$ is nothing but the trial ansatz (2.5) for the zero temperature variational calculation. However, in the present case the kernel $\omega(k)$ is not determined by minimizing the energy but at the moment an arbitrary kernel.

We cannot treat the full density operator, Eq. (3.1). Therefore we replace in the density operator the full Hamiltonian (2.4) by a single-particle one

$$
D=\exp (-\beta h), \quad h=\int \mathrm{d} p \varepsilon(p) a^{\dagger}(p) a(p) .
$$

As a consequence, for the thermal averages (3.2) Wick's theorem applies, which tremendously simplifies the calculations of thermal expectation values. With the density matrix (3.7) one finds the usual Bose occupation numbers

$$
\left\langle a^{\dagger}(p) a(p)\right\rangle \equiv n(p)=(\exp (\beta \varepsilon(p))-1)^{-1},
$$

while the finite-temperature gluon propagator is given by

$$
\langle A A\rangle_{T}=(1+2 n) /(2 \omega) .
$$


It differs from the zero-temperature propagator (2.6) only by the presence of the finite-temperature occupation numbers $n(p)$. In the limit of a vanishing temperature $T \rightarrow 0$ the $n(p)$ vanish and Eq. (3.9) reduces to the zero-temperature propagator, Eq. (2.6).

With the density operator (3.7) at hand, we can straightforwardly calculate the entropy

$$
S=-k_{B} \operatorname{Tr} D \ln D
$$

and the free energy

$$
F[\varepsilon, \omega]=\langle H\rangle_{T}-T S .
$$

A comment is here in order: We cannot calculate in here the free energy from the partition function $Z=\operatorname{Tr} D$ since the density matrix $D$ (3.7) is not yet known. Rather we calculate the free energy by taking the thermal expectation value of the full Hamiltonian. This has in addition the advantage that also two-body correlations are included, which cannot be captured by the single-particle density matrix (3.7).

\section{The deconfinement phase transition}

So far, we have two unknown kernels. The single-particle energies $\varepsilon(p)$ occurring in the single-particle density operator (3.7) and the kernel $\omega(p)$ occurring in the vacuum state (3.6) of our Fock basis (3.4), (3.6). We now determine these kernels by minimizing the free energy (3.11). Since $n(p)$ is a monotonous function of $\varepsilon(p)$ we can vary $F[\varepsilon, n]$ with respect to $n(p)$, which yields the relation [11]

$$
\varepsilon(p)=\omega(p) .
$$

This relation is not surprising given the form of the thermal gluon propagator (3.9), which identifies $\omega(p)$ as quasi-gluon energy. Let us stress, however, that if one goes beyond the present approximation and includes also the Coulomb term (2.4), one obtains still a linear relation between $\varepsilon(p)$ and $\omega(p)$, however, the proportionality factor differs from one by an additional loop integral [10]. Finally, variation with respect to the choice of our basis, i.e. with respect to $\omega(p)$ yields the gap equation

$$
\omega^{2}(p)=p^{2}+\chi^{2}(p)+I[n],
$$

where $\chi(p)$ denotes the ghost loop calculated from the finite-temperature gluon propagator and $I[n]$ denotes the tadpole of the transversal spatial gluons, see Fig. 2. The gap equation (4.2) has to be solved together with the Dyson-Schwinger equation for the ghost form factor $d(p)$. These equations can be solved analytically in the infrared by power law ansätze

$$
\omega(p)=\frac{A}{p^{\alpha}}, \quad d(p)=\frac{B}{p^{\beta}} .
$$

Assuming the horizon condition $d^{-1}(p=0)=0$ one finds at zero temperature from the ghost DSE the sum rule

$$
\alpha=2 \beta+2-d
$$




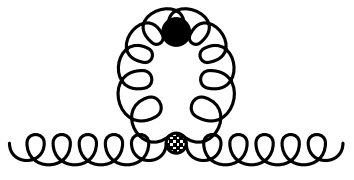

Figure 2: Tadpole term $I[n]$.

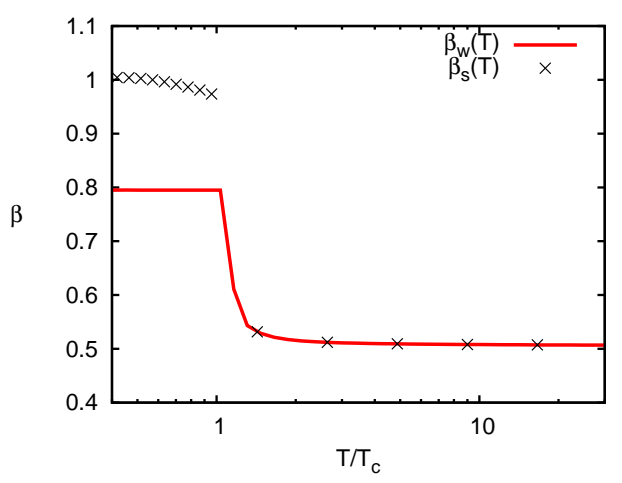

Figure 3: Infrared exponent $\beta$ of the ghost form factor as a function of temperature.

where $d$ is the number of spatial dimensions. With this sum rule one finds then from the gap equation the following solutions for the infrared exponent $\beta$

$$
\begin{array}{ll}
d=3: & \beta=1.00, \quad \beta \approx 0.795 \\
d=2: & \beta=0.50 .
\end{array}
$$

The same exponents are extracted numerically from the self-consistent solution of the equations of motion.

At arbitrarily finite temperature an infrared analysis is impossible due to the fact that the gluon energy $\omega(p)$ occurs in the occupation number (3.8) in exponential form. One can, however, carry out the infrared analysis at infinite temperature, where the Bose occupation numbers given by Eqs. (3.8), (4.1) reduce to

$$
T \rightarrow \infty: \quad n(p) \rightarrow(\beta \omega(p))^{-1} .
$$

One finds then the same sum rule (4.4) as at zero temperature and a single solution with

$$
T \rightarrow \infty: \quad d=3: \quad \beta=0.5, \quad \alpha=0 .
$$

Figure 3 shows the infrared exponent of the ghost form factor $\beta$ as function of temperature. At low temperature one finds the two solutions for the infrared exponents obtained from the zerotemperature infrared analysis. These exponents stay more or less constant as the temperature increases up to a critical temperature, where the two solutions for the infrared exponent $\beta$ merge to a single one, which with increasing temperature approaches the value $\beta=\frac{1}{2}$ predicted by the infrared analysis in the high temperature limit. Figure 4 shows the numerical solution for the ghost form factor $d(p)$ and the gluon energy $\omega(p)$ for various temperatures. The obtained results are in agreement with the predictions of the infrared analysis. At zero temperature both $d(p)$ and $\omega(p)$ are infrared diverging. Above $T_{c}$ the ghost form factor is still infrared diverging but its infrared exponent is halved while the gluon energy becomes infrared finite in accordance with the prediction of the infrared analysis (4.7). Furthermore above $T_{c}$ the plateau value of the gluon energy in the infrared increases with the temperature. This is seen in Fig. 5, where we show the gluon energy at an infrared cut-off $\lambda_{I R} . \omega\left(\lambda_{I R}\right)=m(T)$ jumps at the deconfinement phase transition from a very 

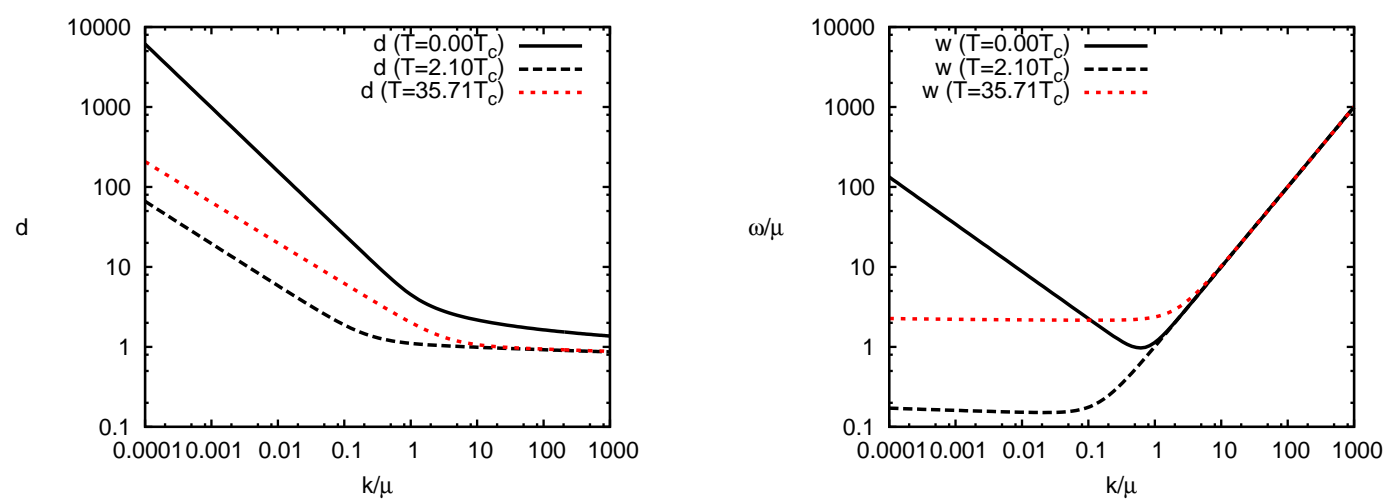

Figure 4: Zero- and finite-temperature solutions for the ghost form factor $d(p)$ (left panel) and the gluon kernel $\omega(p)$ (right panel).

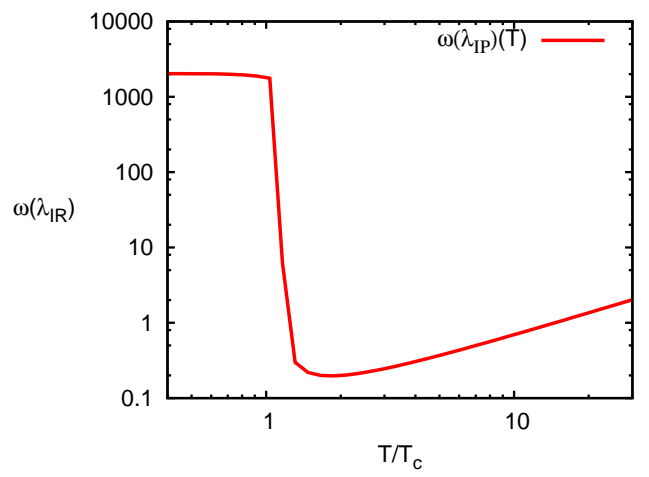

Figure 5: The effective gluon mass $\omega\left(\lambda_{I R}\right)$ as a function of temperature.

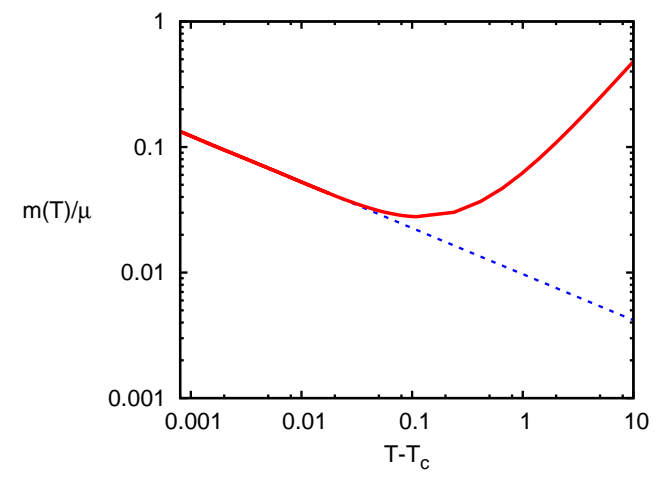

Figure 6: Critical behavior of the effective gluon mass for $T \rightarrow T_{c}$.

large to a small value and afterwards increases linearly with the temperature. Zooming into the transition regime, which is done in Fig. 6 one can extract the critical exponent of $\omega\left(\lambda_{I R}\right)=m(T)$ defined by

$$
m_{\text {crit }}(T) \sim\left(T / T_{C}-1\right)^{\kappa}
$$

and obtains the value $\kappa=0.37$. This value compares well with the value $\kappa=0.41$ obtained in Ref. [12] in a phenomenological quasi-particle model for the gluons using input data from the $d=3$ Ising model, which is in the same universality class as $\mathrm{SU}(2)$ gauge theory. Fitting our scale at the value of the Gribov mass $M=0.88 \mathrm{GeV}$, see Eq. (2.7), determined in Ref. [13] on the lattice, we find a critical temperature for the deconfinement phase transition of $T_{c}=275 \ldots 290 \mathrm{MeV}$. This is in reasonable agreement with the lattice value of $T_{c}=295 \mathrm{MeV}$. This result is also in reasonable agreement with the value $T_{c} \simeq 270 \mathrm{MeV}$ obtained from the effective potential of the Polyakov loop calculated in Ref. [14] within the Hamiltonian approach. Let me also mention that recently the Hamilton approach to Yang-Mills theory in Coulomb gauge has been also used to study glueballs at finite temperature [15]. The results obtained in the gluon sector at finite temperature are quite encouraging for an extension of the Hamilton approach to full QCD at finite temperature and baryon density. 


\section{References}

[1] C. Feuchter and H. Reinhardt, Phys. Rev. D 70, 105021 (2004), hep-th/0402106.

[2] D. Epple, H. Reinhardt, and W. Schleifenbaum, Phys. Rev. D 75, 045011 (2007).

[3] W. Schleifenbaum, M. Leder, and H. Reinhardt, Phys. Rev. D 73, 125019 (2006).

[4] H. Reinhardt and D. Epple, Phys. Rev. D 76, 065015 (2007).

[5] M. Pak and H. Reinhardt, Phys. Rev. D 80, 125022 (2009).

[6] D. R. Campagnari and H. Reinhardt, Phys. Rev. D 78085001 (2008).

[7] D. Schutte, Phys. Rev. D 31, 810 (1985).

[8] A. P. Szczepaniak and E. S. Swanson, Phys. Rev. D 65, 025012 (2002).

[9] D. R. Campagnari and H. Reinhardt, Phys. Rev. D 82, 105021 (2010).

[10] H. Reinhardt, D. R. Campagnari and A. P. Szczepaniak, Phys. Rev. D 84, 045006 (2011).

[11] J. Heffner, H. Reinhardt and D. R. Campagnari, Phys. Rev. D 85, 125029 (2012).

[12] P. Castorina, D. E. Miller, H. Satz, Eur. Phys. J. C 71, 1673 (2011).

[13] G. Burgio, M. Quandt, H. Reinhardt, Phys. Rev. Lett. 102, 032002 (2009).

[14] H. Reinhardt and J. Heffner, Phys. Lett. B 718, 2 (2012).

[15] T. Yepez-Martinez, A. P. Szczepaniak and H. Reinhardt, Phys. Rev. D 86, 076010 (2012). 